\title{
DNA Content of Mitotically-active Condensed Chromosomes of Drosophila melanogaster*
}

\author{
H. Gay, C. C. Das, K. Forward, and B. P. KaUfinanN \\ Department of Zoology and Cytogenetics Laboratory of Carnegie Institution \\ of Washington, The University of Michigan, Ann Arbor, Michigan \\ Received October 7, 1970 / Accepted October 11, 1970
}

Abstract. Two-wavelength Feulgen microspectrophotometry was used to determine the DNA content of mitotically-active ganglionic cells of first- and thirdinstar larvae of Drosophila melanogaster. The measurements revealed that the DNA values differ, on the average, by a factor of approximately two, with the metaphase cells of the first-instar larvae having about four times the haploid amount of the spermatozoon, and the metaphase cells of the third-instar larvae having about eight times the haploid amount. The increase from $4 \mathrm{C}$ to $8 \mathrm{C}$ in the course of development without any pronounced modification of the heterochromatic-euchromatic ratio is interpreted as evidence of an increase in the number of chromosomal strands. It is suggested, accordingly, that these mitotically-active chromosomes are multistranded or polynemic.

\section{Introduction}

The condensed metaphase and anaphase chromosomes of ganglionic cells of first-instar larvae of Drosophila melanogaster are in general perceptibly smaller than those of third-instar larvae (Fig. 1). The difference in size could conceivably be due to a difference in either the amount of nucleic acids or the amount of proteins. With respect to DNA, specific information about the quantity of this nucleic acid in a given chromosome complex can be obtained by application of microspectrophotometric methods. An ultraviolet-photometric study by Rudkin (1963) suggested that the ganglionic metaphase chromosomes of larvae of $D$. melanogaster shortly after hatching contain approximately onefourth the amount of DNA that is present in metaphase plates obtained from late third-instar larvae, although both have the normal chromosome complement of the strain and the normal distribution between heterochromatic and euchromatic regions. Using two-wavelength Feulgen microspectrophotometry, Swift (1962) found differences in DNA quantities in interphase nuclei of cerebral ganglion cells in late third-instar

* This study was supported by a Research Grant (GM 10499) from the National Institutes of Health, U.S. Public Health Service. 
larvae of Drosophila virilis, which he attributed to polyteny. Chromosomes in early polytenic stages of development were also detected cytophotometrically in the brain ganglion of Drosophila hydei by Berendes and Keyl (1967). Similar evidence of endoreduplication during development has been obtained in studies of other genera of insects (for example, in a densitometric analysis by Fox, $1969 \mathrm{~b}$, of abdominal fat body and testis-wall cells in seven species of the beetle, Dermestes).

To gain further information about the differences in DNA content of ganglion cells of $D$. melanogaster, we carried out two-wavelength Feulgen microspectrophotometry of metaphase (and a few anaphase) plates of first- and third-instar larvae. Since the measurements were made on well spread chromosome complexes of actively dividing cells where the $2 n$ number of eight could be determined readily, any increase in DNA would presumably reflect either an increase in the amount of this nucleic acid per chromosomal strand, or a difference in the number of strands. Our findings reveal that actively dividing neuroblasts of $D$. melanogaster have chromosome complements that vary appreciably in their DNA content without pronounced modification of the heterochromaticeuchromatic ratio. It is suggested, therefore, that this situation reflects a difference in the number of strands composing the mitotic chromosome. Preliminary reports of these findings have been given by Gay $(1964,1965,1966)$.

\section{Materials and Methods}

All observations were made on preparations obtained from the wild-type Swedish-b strain of Drosophila melanogaster. Healthy adult females were allowed to lay eggs on an agar-dextrose-cornmeal-yeast medium that had been poured into small petri dishes. The dishes were fastened onto widemouthed bottles during the period of egg laying, but were then removed and covered, after the food had been enriched with yeast to ensure development of large well-fed larvae.

First-instar larvae, which were carefully timed at the moment of hatching, were sacrificed after 16 hours of development at $25^{\circ} \mathrm{C}$. Petri dishes with thirdinstar larvae were transferred to a $17^{\circ} \mathrm{C}$ chamber at the time of the second molt, where they remained until the larvae were sacrificed an hour or two before the time of eversion of the anterior spiracles that marks the end of the third instar. In a few cases before the first- or third-instar larvae were sacrificed they were fed for two to three hours with a yeast-sugar solution containing 0.05 to 0.1 percent colchicine in an effort to increase the number of analyzable metaphase figures.

Using tungsten needles an entire cerebral ganglion was dissected from a female larva and held for 10 minutes in one per-cent hypotonic sodium citrate solution. The ganglion was then fixed. In most cases, it was transferred to a drop of 50 per-cent acetic acid on a gelatin chromalum subbed slide, where it remained for 15 minutes before a cover slip was attached and the preparation was squashed by thumb pressure (the slide being placed between layers of paper toweling to absorb the excess fluid). The preparation was then placed immediately on solid $\mathrm{CO}_{2}$ (dry ice), where it remained for about 30 minutes before the cover slip was flipped off. The slides were then defrosted in ice cold absolute alcohol. After hydration all 
slides were rinsed in distilled water, hydrolyzed for 14 minutes in $1 \mathrm{~N} \mathrm{HCl}$ at $60^{\circ} \mathrm{C}$, rinsed again in distilled water, and then stained for two hours in the dark with Schiff's solution to form the Feulgen reaction. After three changes of 10 minutes each in bleaching solution, the slides were dehydrated and mounted in Cargille's liquid having a refractive index of 1.540. In these staining procedures all slides were handled in the same glass rack so that the tissues were exposed to the same solutions simultaneously and for the same length of time. A slight modification of the procedure listed above was used on the ganglia obtained from the larvae that were fed colchicine. The brains were dissected in one percent hypotonic sodium citrate solution and then fixed in formol-acetic acid rather than in acetic acid alone. They were hydrolyzed for 35 minutes in $5 \mathrm{~N} \mathrm{HCl}$ at room temperature and then stained by the Feulgen reaction. The stained glands were squashed and the cover slips were removed by the dry-ice technique. The preparations were then dehydrated and mounted in Cargille's liquid.

In order to make a quantitative determination of the haploid (1C) amount of DNA in D. melanogaster, measurements were made of the mature spermatozoa. Testes were obtained from males that had been separated from females for five days. The posterior regions of the testes near the testicular ducts were dissected and placed in 50 per-cent acetic acid on subbed slides for 10 minutes, and then gently squashed. Following dry-ice treatment, the preparations were stained by the Feulgen method and then mounted for microscopical study as outlined above.

A Pollister-type instrument was employed in our two-wavelength studies. The microspectrophotometer uses a $250 \mathrm{~mm}$ Bausch and Lomb grating monochromator in conjunction with a Bausch and Lomb compound microscope equipped with a spectrophotometer of our own design. Magnification was achieved with the compound microscope by using either a $2 \times$ or $5 \times$ ocular and either a $43 \times$ or $90 \times$ oil immersion objective. The light measuring device consists of an RCA 1 P21 phototube connected to an RCA ultrasensitive microammeter through a power supply and control unit made by the Farrand Optical Company. Before any measurements were made, the equipment was checked for response in respect to area, and was found to maintain linearity of current output with increasing incident light up to a diameter of $11.0 \mathrm{~mm}$, the effective aperture of the phototube, for a series of different wavelengths in several regions of the spectrum.

The microspectrophotometric technique used was based on the two-wavelength method described by Patau (1952). The two wavelengths $\lambda_{1}$ and $\lambda_{2}$ were determined from absorption curves of the tissue measured. For the bulk of the material these wavelengths were $560 \mathrm{~m} \mu$ and $498 \mathrm{~m} \mu$; for the smaller colchicine-treated fraction the wavelengths were $570 \mathrm{~m} \mu$ and $500 \mathrm{~m} \mu$. Under these circumstances, the extinction values stand in a 1 to 2 relationship $\left(E \lambda_{1}=2 E \lambda_{2}\right)$. Reliability of the measurements was further confirmed by comparing transmission data of three neutral density filters and a Wratten No. 66 filter with the data provided by a Beckman spectrophotometer. Confirmation of the validity of the two-wavelength method for quantitative determination of amounts of DNA in condensed chromosomes was obtained by a spectrophotometric comparison of Feulgen-stained roottip cells of Tradescantia paludosa in different stages of mitosis. Every measurement, which is reported in arbitrary units, is the mean of two independent readings taken on a given configuration.

\section{Results}

Measurements of the amount of DNA in the spermatozoon were obtained by readings made on five groups containing respectively 25 , 

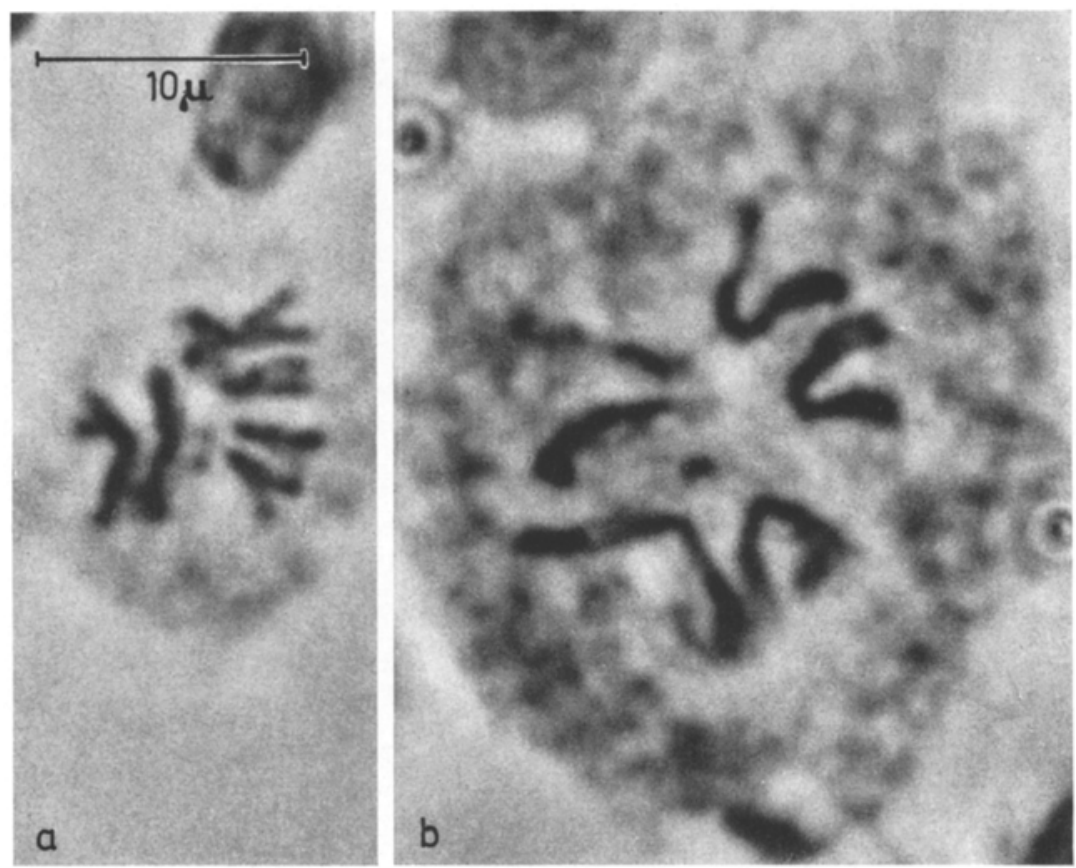

Fig. $1 \mathrm{a}$ and b. Metaphase ganglionic cells. Phase contrast photograph. $\times 2750$. a Metaphase from first instar larva. b Metaphase from third-instar larva

20, 18, 15, and 9 spermatozoa. From these readings a haploid or $1 \mathrm{C}$ value of $27 \pm 1.18$ arbitrary units was derived.

Preliminary measurements on seven first-instar ganglionic (neuroblast) metaphase plates, obtained in the colchicine-treated series, showed a mean amount of DNA in arbitrary units of 105.1 \pm 5.85 . In eleven third-instar neuroblast metaphase plates, from the same series, the mean amount in arbitrary units was $200.7 \pm 11.01$. Application of the $t$ test to these values showed that we were unquestionably dealing with two distinct populations of cells. In them the DNA values differ by a factor of approximately two, and are respectively about four times and eight times the sperm values (assumedly revealing $4 \mathrm{C}$ and $8 \mathrm{C}$ metaphase plates).

Additional measurements on eight first-instar ganglionic metaphase plates, from the non-colchicine treated series, disclosed a mean amount of DNA in arbitrary units of $104.1 \pm 4.64$. This value is not significantly different from that of the colchicine-treated series. In 28 third-instar neuroblast plates, from the non-colchicine treated series, the mean amount in arbitrary units was $190.7 \pm 8.13$. This value is similar to that 


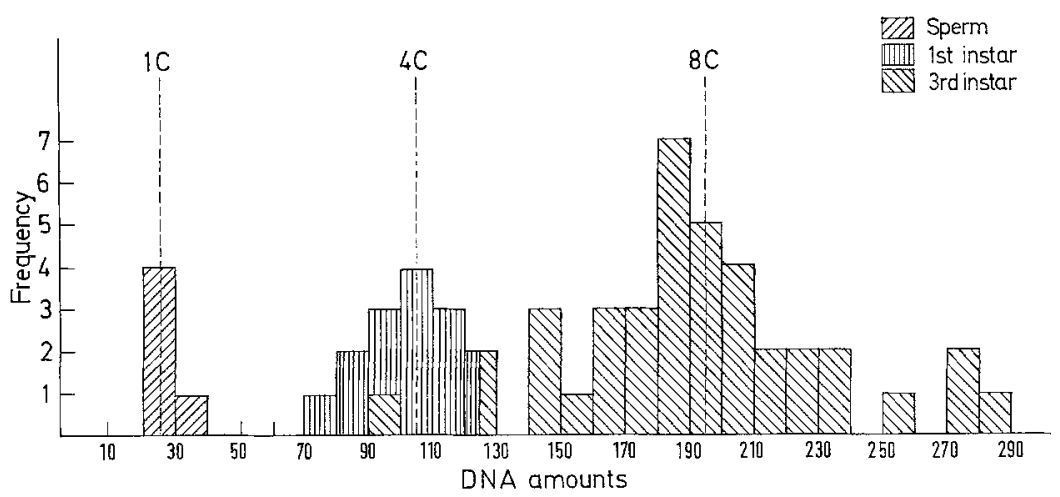

Fig. 2. Histogram showing amounts of DNA measured in 5 groups of spermatozoa $(1 C)$, I5 first-instar ganglionic metaphase plates $(4 C)$, and 39 third-instar ganglionic metaphase plates $(8 C)$. Fuller explanation in text

obtained from the colchicine-treated third-instar metaphase complexes. When the values for the first- and third-instar classes of the noncolchicine treated material were subjected to statistical comparison they were found to be different at the 99 per-cent confidence level. This suggests again that we were measuring for the most part metaphase complexes that fall into either the $4 \mathrm{C}$ or $8 \mathrm{C}$ category.

Because of the statistically-confirmed similarity of the colchicinetreated and untreated materials, all of the data have been combined. Measurements on the 15 first-instar ganglionic metaphase plates showed a mean amount of DNA in arbitrary units of $104.6 \pm 3.69$; and measurements on 39 third-instar metaphase plates showed a mean amount of $193.5 \pm 6.65$ arbitrary DNA units. The distributions of the different classes are plotted in the histogram presented as Fig. 2. It is apparent that the ganglion cells from third-instar larvae contain about twice as much DNA as those from first-instar larvae. Assumedly the metaphases with the lower DNA values contain the diploid (4C) amount for $D$. melanogaster and most of those with the higher values contain the tetraploid (8C) amount.

It might be assumed that these $8 \mathrm{C}$ metaphase complexes contain diplochromosomes, composed of four chromatids loosely held together by a single centromere; but they would then be expected to fall apart at anaphase to form a tetraploid cell. We have not observed any mitotic figures with more than the normal diploid number, but as a further check we have examined microspectrophotometrically seven third-instar anaphase figures in which the diploid chromosome number of eight could readily be determined. Each measurement included the two sets 
of sister chromosomes directed toward the spindle poles. From these measurements the mean amount of DNA in arbitrary units was determined as $204.0 \pm 25.14$. One of the anaphase groups contained 311 arbitrary units. This value is larger than any found in our study of third-instar metaphases and suggests that we might be measuring a cell that belongs to the $16 \mathrm{C}$ class. If so, we would be detecting a thirdinstar cell that contains four times the amount of DNA characteristic of the first-instar larva and would be confirming the relationship suggested by Rudkin (1963). The results in general indicate that the doubled DNA values observed for metaphase plates are also found in anaphase figures. Thus the production of diplochromosomes does not account for the $8 \mathrm{C}$ amounts of DNA found in third-instar larval metaphase plates.

\section{Discussion}

Two-wavelength Feulgen microspectrophotometry has shown that third-instar larval ganglionic cells of Drosophila melanogaster have, on the average, twice as much DNA as first-instar ganglionic cells. The reliability of these measurements has been confirmed statistically by comparison of the means and standard errors, and by application of the $t$ test and analysis of variance. The statistical analysis has supported the assumption that the metaphase ganglion cells of the first-instar larvae contain the $4 \mathrm{C}$ amount of DNA whereas the same kind of cells in the third-instar larvae are in general $8 \mathrm{C}$. If the haploid (1C) sperm value is multiplied by eight, and the first-instar metaphase value is multiplied by two, and these are compared by analysis of variance with the measured metaphase values for the third instar, no significant difference is found, since the $\mathrm{F}$ ratio is 0.7217 . However, the measurements made on first-instar cells are more uniform than those made on third-instar cells. This result suggests that the first-instar population of cells is relatively homogeneous and that errors of spectrophotometry are minimal. On the other hand, the third-instar population appears to be more heterogeneous, having some $4 \mathrm{C}$ and an occasional $16 \mathrm{C}$ cell in addition to the predominantly $8 \mathrm{C}$ group of cells. Assessment of all the statistical analyses shows, however, that errors of spectrophotometry are not significantly greater when strongly condensed chromosomes are more widely separated in a given field. The over-all evaluation clearly demonstrates that the chromosomes of ganglion cells from thirdinstar larvae of $D$. melanogaster have, on the average, twice as much DNA as the ganglion cells from first-instar larvae.

Detection of a two-fold difference in the amount of deoxyribonucleic acid in first- and third-instar ganglionic cells raises the important question whether the increase from $4 \mathrm{C}$ to $8 \mathrm{C}$ during ontogeny is 
attributable to longitudinal repetitiveness or to a lateral increase in the number of strands per chromosome. As is indicated by comparison of Fig. $1 \mathrm{a}$ and $\mathrm{b}$, the chromosomes during third instar are longer and wider than they are during first instar. A two- to four-fold difference in chromosomal volume is usually discernible. Therefore, gross examination of condensed chromosomes does not serve to discriminate satisfactorily between the two alternative possibilities of the method of increase in amounts of DNA. Inspection of late prophase chromosomes offers some relevant information, since the heterochromatic regions adjacent to the centromeres seem to represent the same proportion of the total length of the chromosomes in all the first- and third-instar cells examined (cf. Rudkin, 1963).

Evidence on a gross scale in Drosophila melanogaster for localized longitudinal repetitiveness of chromosomal DNA is presented by tandem duplications such as those that accompany the Bar and Hairy-wing phenotypes. On a molecular scale in this species, hybridization studies by Ritossa and Spiegelman (1965) and Ritossa, Atwood, and Spiegelman (1966) have shown that the r-DNA ("ribosomal" DNA, which forms molecular hybrids with ribosomal RNA) is highly redundant and is associated with the nucleolus organizer. Variations that occur in the redundancy of $\mathrm{r}$-DNA can be explained on the assumption that the DNA templates are present in the organizer as tandem repeats. Fvidence for the occurrence of longitudinal accretion in somatic cells of another dipteran has been presented by Keyl (1965a, b) in a comparative study of two subspecies of Chironomus. When the DNA content was determined in certain salivary-gland-chromosome bands of C.thummi thummi and $C$.th. piger, a geometric series of $1: 2,1: 4,1: 8$, and $1: 16$ was detected. These changes were not confined to the salivary-gland chromosomes, since C. th. thummi has 27 per cent more DNA than C.th. piger in its gametes as well as in its salivary-gland chromosomes.

Further inferences of the existence of high degrees of longitudinal repetitiveness were derived from studies of the lamplorush chromosomes of amphibians. Gall (1963) has interpreted the kinetics of the degradational action of deoxyribonuclease as evidence that the chromatid consists of a single Watson-Crick double helix. This interpretation is consonant, moreover, with the observation that the chromosome replicates semiconservatively (Taylor, 1963).

Since recombination data indicate that each gene is represented only once per chromatid and that different genes are contiguous, Whitehouse (1967) has suggested that the chromosome has the form of a cycloid, each loop of which wonld correspond to a set of copies of a gene forming a chromomere. Accordingly, at meiosis the redundant copies of the gene 
are assumedly detached to form a single circular DNA molecule. The remaining master copy would then be in a position to undergo crossingover with a homologous chromatid. Subsequently, the duplicate copies could conceivably be restored to the chromatid by crossing-over between one of their number and the master copy. As visualized, the chromosome can alternate between two states with each set of duplicate genes detached as a circle or integrated with the main DNA axis. Although the model assumes that the repeating sequences are identical, the reassociation studies of Britten and Kohne (1968) suggest that groups of repeated sequences often consist of similar rather than identical members. Moreover, the chromosome model envisioned by Uhl (1965) reconciles high degrees of lateral multiplicity with semiconservative replication. Such a polynemic chromosome would consist of several to many parallel double helices connected in groups to functionally single links. Due allowance is thus made for those cases of redundancy that represent multiples of the entire genome. Rothfels et al. (1966), for example, have offered the interpretation of polynemy to account for the differences between 22 diploid species of Ranunculaceae whose DNA values form a nongeometric series with the terms $1,8,12,16,20,24$, and 40. Additional evidence of differences in amount of DNA among mitotically-active cells was obtained in cytophotometric studies of Pinus silvestris by Nagl (1967), who suggested that the differences might be attributable to varying numbers of strands per chromosome. On the other hand, Fox (1969a) made a comparative study of six species of Dermestes, using scanning densitometry of Feulgen-stained spermatids, and found that the DNA values covered a 2.7-fold range. Since the distribution does not represent a geometric series, Fox concludes that the differences do not reflect a variation in strand number.

In the unineme concept of Whitehouse - and the somewhat similar proposal of Callan (1967) - the length of the replicating DNA strand would increase proportionately as the ganglionic cells of $D$. melanogaster increased their total DNA content from $4 \mathrm{C}$ to $8 \mathrm{C}$ and then to $16 \mathrm{C}$. On the other hand, the polyneme concept would attribute redundancy to a lateral increase in number of intact chromosomal strands. This is the accepted explanation of the high multiples of DNA that characterize the salivary-gland chromosomes of Diptera. With the lower multiples the chromosomes may retain their capacity for mitotic activity, whereas at the higher multiples the component strands aggregate to form a polytenic complex. Nittwoch et al. (1966) inferred from microphotometric examination of Feulgen-stained nuclei of drone and worker larvae of the honey bee that dividing cells tend to retain the diploid chromosome number but that a portion of the cells become polyploid in interphase. An earlier microspectrophotometric study by Merriam and Ris (1954) 
had shown that polyploidization occurs in the somatic cells of both male and female honey bees.

As noted by Wolff (1969), observational, experimental, and inferential types of evidence that chromosomes can be multistranded have accumulated over the years. One of the most compelling lines of observational support was afforded by the $16 \mathrm{~mm}$ time-lapse micrographic technique, which revealed anaphasic duality (half chromatids) in chromosomes of living endosperm of Haemanthus (Bajer, 1965). Observations of fixed and stained ganglionic cells of $D$. melanogaster by Kaufmann (1934) also revealed that "anaphase chromosomes are longitudinally double, the split following the turns of the chromonemata". These were from ganglia of third-instar larvae and presumably represented $8 \mathrm{C}$ cells. The split separating half chromatids testifies to the polynemic nature of these chromosomes. Although much of the evidence for polynemy has been obtained in studies of plant chromosomes (see Kaufmann, Gay and McDonald, 1960; Wolff, 1969 for reviews), the results obtained in the present study of Drosophila melanogaster present further evidence that changes in the degree of polynemy can occur in the course of development of an organism. The selective advantages afforded by the presence of multiple copies of genes are attained in some cases by development of polyploidy or polyteny, whereas in others-such as the neuroblasts of $D$. melanogaster-polynemy ensues. As stated by Holliday (1970), the evidence for both longitudinal and lateral multiplicity within chromosomes of eukaryotes now seems very strong, and it may well be that special mechanisms have been developed for the maintenance and replication of such chromosomes. The nature of these mechanisms thus becomes a major problem for investigations in the future. And, as noted by Kaufmann, Gay, and MoDonald, the discovery of subchromonemata within each chromatid does not pose a problem that is new in principle, but does emphasize the need for a solution in terms of conditions that can mold a large group of strands into a functionally single structure. The organizational patterns within chromosomes of eukaryotes present an outstanding challenge to eytogeneticists in their continuing efforts to shed new light on problems of cellular metabolism, growth, and differentiation.

\section{References}

Bajer, A.: Subchromatid structure of chromosomes in the living cell. Chromosoma (Berl.) 17, 291-302 (1965).

Berendes, H. D., Keyl, H.-G.: Distribution of DNA in heterochromatin and euchromatin of polytene nuclei of Drosophila hydei. Genetics 57, 1-13 (1967).

Britten, R. J., Kohne, D. E.: Repeated sequences in DNA. Science 161, 529-540 (1968). 
Callan, H. G.: The organization of genetic units in chromosomes. J. Cell Sci. 2, $1-7(1967)$.

Fox, D. P.: The relationship between DNA value and chromosome volume in the coleopteran genus Dermestes. Chromosoma (Berl.) 27, 130-144 (1969a).

- DNA values in somatic tissues of Dermestes (Dermestidae: Coleoptera) I. Abdominal fat body and testis wall of the adult. Chromosoma (Berl.) 28, 445-456 $(1969 \mathrm{~b})$.

Gall, J. G.: Kinetics of deoxyribonuclease action on chromosomes. Nature (Lond.) $198,36-38$ (1964).

Gay, H.: Chromosome structure and function. Carnegie Inst. Wash. Year Book 68, 605-614 (1964).

- Organization of chromosomes in higher forms. Carnegie Inst. Wash. Year Book 64, 539-614 (1965).

- Chromosome organization in eucaryotes. Carnegie Inst. Wash. Year Book 65, $581-587(1966)$.

Holliday, R.: The organization of DNA in eukaryotic chromosomes. In: Organization and Control in Prokaryotic and Eukaryotic Cells, 20th Symp. Soc. gen. Microbiol. (H. P. Charles and B. C. J. G. Knight, eds.), p. 359-380. New York: Cambridge University Press 1970.

Kaufmann, B. P.: Somatic mitoses of Drosophila melanogaster J. Morph. 56, 125155 (1934).

- Gay, H., McDonald, M. R.: Organizational patterns within chromosomes. In: International review cytology, vol. 9 (G. H. Bourne and J. G. Danielli, eds.), p. 77-127. New York: Academic Press 1960.

Keyl, H.-G.: A demonstrable local and geometric increase in the chromosomal DNA of Chironomus. Experientia (Basel) 21, 191-193 (1965a).

- Duplikationen von Untereinheiten der chromosomalen DNA während der Evolution von Chironomus thummi. Chromosoma (Berl.) 17, 139-180 (1965b).

Merriam, R.W., Ris, H.: Size and DNA content of nuclei in various tissues of male, female, and worker honey bees. Chromosoma (Berl.) 6, 522-538 (1954).

Mittwoch, U., Kalmus, H., Webster, W. S. : Deoxyribonucleic acid values in dividing and non-dividing cells of male and female larvae of the honey bee. Nature (Lond.) 210, 264-266 (1966).

Nagl, W.: Mikrophotometrische DNA-Messungen an Interphase- und Ruhekernen sowie Mitosen in der Samenanlage von Pinus silvestris. Z. Pflanzenphysiol. 56, 40-56 (1967).

Patau, K.: Absorption microphotometry of irregular-shaped objects. Chromosoma (Berl.) อ, 341-362 (1952).

Ritossa, F. M., Spiegelman, S.: Localization of DNA complementary to ribosomal RNA in the nucleolus organizer region of Drosophila melanogaster. Proc. nat. Acad. Sci. (Wash.) 53, 737-745 (1965).

Rothfels, K., Sexsmith, E., Heimburger, M., Krause, M. O.: Chromosome size and DNA content of species of Anemone $L$. and related genera (Ranunculaceae). Chromosoma (Berl.) 20, 54-74 (1966).

Rudkin, G. T.: The structure and function of heterochromatin. In: Genetics today, vol. 2 (S. J. Geerts, ed.), p. 359-374. Oxford: Pergamon Press 1963.

Swift, H.: Nucleic acids and cell morphology in dipteran salivary glands. In: The molecular control of cellular activity (J. M. Allen, ed.), p. 73-125. New York: MeGraw-Hill Book Co. 1962. 
Taylor, J.H.: The replication and organization of DNA in chromosomes. In: Molecular genetics, part 1 (J. H. Taylor, ed.), p. 65-111. New York: Academie Press 1963.

Uhl, C. H.: Chromosome structure and crossing over. Genetics 51, 191-207 (1965). Whitehouse, H. L. K.: A cycloid model for the chromosome. J. Cell Sci. 2, 9-22 (1967).

Wolff, S.: Strandedness of chromosomes. In: Intern. Rev. Cytol., vol. 25 (G. H. Bourne and J. G. Danielli, eds.), p. 279-296. New York: Academic Press 1969 .

Professor B. P. Kaufmann

Department of Zoology

The University of Michigan

Ann Arbor, Mich. 48104

U.S.A. 